\title{
Teaching NeuroImages: Symmetrical abnormalities of the globi pallidi in succinic semialdehyde dehydrogenase deficiency
}

Silvia Esposito, MD, PhD, Marco Moscatelli, MD, Claudio Caccia, PhD, Elisa Granocchio, MD, Chiara Pantaleoni, MD, Anna Ardissone, MD, PhD, Cinzia Gellera, PhD, and Laura Farina, MD Neurology ${ }^{\circledR}$ 2020;95:e2316-e2317. doi:10.1212/WNL.0000000000010367

\section{Figure Globi pallidi T2 symmetrical hyperintensity}
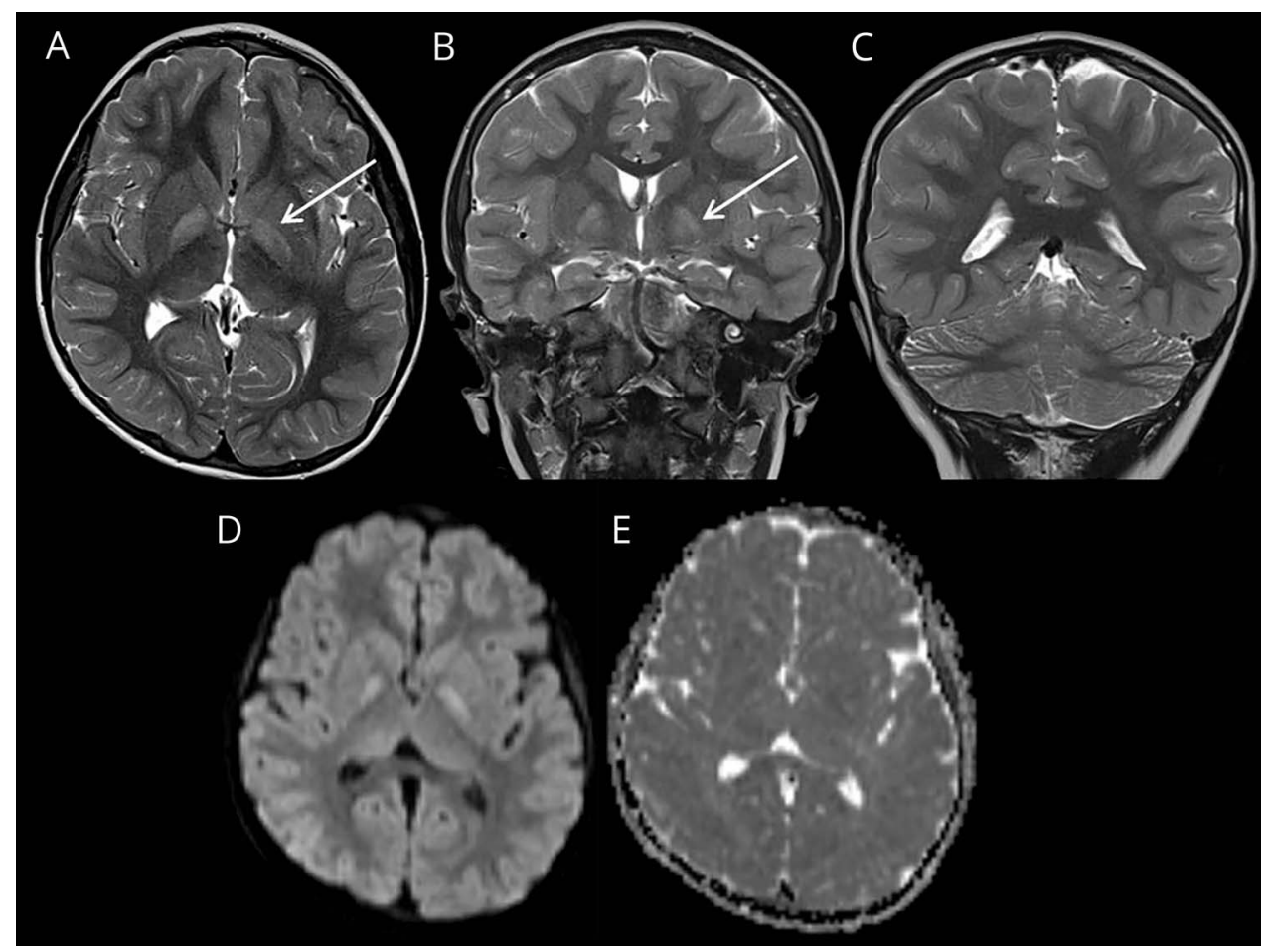

Axial (A) and coronal T2-weighted imaging (B, C) show symmetrical hyperintensity of the globi pallidi (arrows). Note that both pallidal components (internal and external pallidus) are well discernible. No dentate involvement or cerebellar atrophy is seen. (D) Diffusion-weighted images show pallidal diffusion restriction at high b-values confirmed by apparent diffusion coefficient map (E).

A 4-year-old boy born full term after an uneventful pregnancy and with normal early developmental milestones achievement presented hypotonia, motor coordination disorder, and childhood apraxia of speech. Brain MRI revealed globi pallidi T2 symmetrical signal abnormalities (figure). An extensive diagnostic workup panel was performed to exclude metabolic and acquired conditions. ${ }^{1,2}$ Urine organic acid profile showed marked $\gamma$-hydroxybutyrate aciduria $(222 \mu \mathrm{g} / \mathrm{mg}$ creatinine, normal $<5)$. Genetic analysis revealed 2 compound heterozygous pathogenic mutations in the ALDH5A1 gene (p.Pro81Ser, maternal origin; p.Arg527Ter, paternal origin) consistent with succinic semialdehyde
Correspondence

Dr. Esposito

silvia.esposito@

istituto-besta.it 
dehydrogenase deficiency suspicion. This rare entity must be suspected in case of T2-weighted MRI symmetrical abnormalities of the globi pallidi. ${ }^{1,2}$

\section{Study funding}

No targeted funding reported.

\section{Disclosure}

The authors report no disclosures relevant to the manuscript. Go to Neurology.org/N for full disclosures.

\section{Appendix Authors}

\begin{tabular}{|c|c|c|}
\hline Name & Location & Contribution \\
\hline $\begin{array}{l}\text { Silvia } \\
\text { Esposito, } \\
\text { MD, PhD }\end{array}$ & $\begin{array}{l}\text { Fondazione IRCCS Istituto } \\
\text { Neurologico Carlo Besta, } \\
\text { Milan, Italy }\end{array}$ & $\begin{array}{l}\text { Study design and } \\
\text { manuscript writing, care of } \\
\text { the patient }\end{array}$ \\
\hline $\begin{array}{l}\text { Marco } \\
\text { Moscatelli, } \\
\text { MD }\end{array}$ & $\begin{array}{l}\text { Fondazione IRCCS Istituto } \\
\text { Neurologico Carlo Besta, } \\
\text { Milan, Italy }\end{array}$ & $\begin{array}{l}\text { Interpretation of radiologic } \\
\text { images, manuscript } \\
\text { revision for intellectual } \\
\text { content }\end{array}$ \\
\hline $\begin{array}{l}\text { Claudio } \\
\text { Caccia, PhD }\end{array}$ & $\begin{array}{l}\text { Fondazione IRCCS Istituto } \\
\text { Neurologico Carlo Besta, } \\
\text { Milan, Italy }\end{array}$ & $\begin{array}{l}\text { Manuscript revision for } \\
\text { intellectual content, } \\
\text { interpretation of metabolic } \\
\text { and genetic findings }\end{array}$ \\
\hline
\end{tabular}

Appendix (continued)

\begin{tabular}{|c|c|c|}
\hline Name & Location & Contribution \\
\hline $\begin{array}{l}\text { Elisa } \\
\text { Granocchio, } \\
\text { MD }\end{array}$ & $\begin{array}{l}\text { Fondazione IRCCS Istituto } \\
\text { Neurologico Carlo Besta, } \\
\text { Milan, Italy }\end{array}$ & $\begin{array}{l}\text { Manuscript revision for } \\
\text { intellectual content, } \\
\text { interpretation of } \\
\text { neuropsychological } \\
\text { assessment }\end{array}$ \\
\hline $\begin{array}{l}\text { Chiara } \\
\text { Pantaleoni, } \\
\text { MD }\end{array}$ & $\begin{array}{l}\text { Fondazione IRCCS Istituto } \\
\text { Neurologico Carlo Besta, } \\
\text { Milan, Italy }\end{array}$ & $\begin{array}{l}\text { Manuscript revision for } \\
\text { intellectual content }\end{array}$ \\
\hline $\begin{array}{l}\text { Anna } \\
\text { Ardissone, } \\
\text { MD, PhD }\end{array}$ & $\begin{array}{l}\text { Fondazione IRCCS Istituto } \\
\text { Neurologico Carlo Besta, } \\
\text { Milan, Italy }\end{array}$ & $\begin{array}{l}\text { Manuscript revision for } \\
\text { intellectual content, care of } \\
\text { the patient }\end{array}$ \\
\hline $\begin{array}{l}\text { Cinzia } \\
\text { Gellera, PhD }\end{array}$ & $\begin{array}{l}\text { Fondazione IRCCS Istituto } \\
\text { Neurologico Carlo Besta, } \\
\text { Milan, Italy }\end{array}$ & $\begin{array}{l}\text { Manuscript revision for } \\
\text { intellectual content, } \\
\text { interpretation of metabolic } \\
\text { and genetic findings }\end{array}$ \\
\hline $\begin{array}{l}\text { Laura } \\
\text { Farina, MD }\end{array}$ & $\begin{array}{l}\text { Fondazione IRCCS Istituto } \\
\text { Neurologico Carlo Besta, } \\
\text { Milan; Fondazione IRCCS } \\
\text { Fondazione Santa Lucia, } \\
\text { Rome, Italy }\end{array}$ & $\begin{array}{l}\text { Interpretation of radiologic } \\
\text { images, manuscript } \\
\text { revision for intellectual } \\
\text { content }\end{array}$ \\
\hline
\end{tabular}

\section{References}

1. Barkovich AJ. An approach to MRI of metabolic disorders in children. J Neuroradiol 2007;34:75-88.

2. Zuccoli G, Yannes MP, Nardone R, Bailey A, Goldstein A. Bilateral symmetrical basal ganglia and thalamic lesions in children: an update (2015). Neuroradiology 2015;57:973-989. 


\section{Neurology}

\section{Teaching NeuroImages: Symmetrical abnormalities of the globi pallidi in succinic semialdehyde dehydrogenase deficiency}

Silvia Esposito, Marco Moscatelli, Claudio Caccia, et al.

Neurology 2020;95;e2316-e2317 Published Online before print July 17, 2020

DOI 10.1212/WNL.0000000000010367

This information is current as of July 17, 2020

\section{Updated Information \&} Services

References

Subspecialty Collections

Permissions \& Licensing

Reprints including high resolution figures, can be found at: http://n.neurology.org/content/95/16/e2316.full

This article cites 2 articles, 0 of which you can access for free at: http://n.neurology.org/content/95/16/e2316.full\#ref-list-1

This article, along with others on similar topics, appears in the following collection(s):

Developmental disorders

http://n.neurology.org/cgi/collection/developmental_disorders Metabolic disease (inherited)

http://n.neurology.org/cgi/collection/metabolic_disease_inherited MRI

http://n.neurology.org/cgi/collection/mri

Neuropsychological assessment

http://n.neurology.org/cgi/collection/neuropsychological_assessment

Information about reproducing this article in parts (figures,tables) or in its entirety can be found online at:

http://www.neurology.org/about/about_the_journal\#permissions

Information about ordering reprints can be found online:

http://n.neurology.org/subscribers/advertise

Neurology ${ }^{\circledR}$ is the official journal of the American Academy of Neurology. Published continuously since 1951, it is now a weekly with 48 issues per year. Copyright (C 2020 American Academy of Neurology. All rights reserved. Print ISSN: 0028-3878. Online ISSN: 1526-632X.

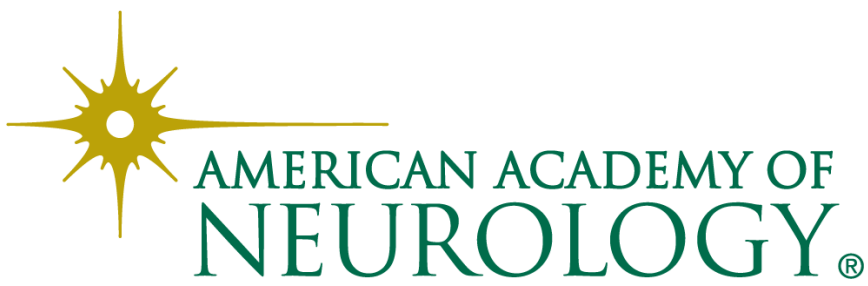

